\title{
Electron Density Building Block approach for Metal Organic Frameworks
}

\author{
Abita S. Chimpri, Piero Macchi* \\ Department of Chemistry and Biochemistry, University of Bern, Freiestrasse 3, CH- \\ 3012 Bern, Switzerland. \\ piero.macchi@dcb.unibe.ch
}

\begin{abstract}
A general introduction is given to the state of the art in modeling metal organic materials using transferable atomic multipoles. The method is based on the building block partitioning of the electron density, which is illustrated with some examples of the potential applications and with detailed discussion of the necessities and pitfalls. The interactions taking place between building blocks are summarized and used to discuss the properties that can be calculated.
\end{abstract}

\section{Introduction}

Metal organic materials, in particular metal organic frameworks [1] (MOF), have attracted the attention of material scientists and chemists in the past three decades, thanks especially to their tunable properties, the simple fabrication processes and the wide range of applications and functionalities [2]. These materials are based on three kinds of building blocks (BB): organic ligands (usually called linkers), metallic centers (connectors) and extra-framework molecules or counter-ions (guests). The linkers are neutral or anionic species, the connectors are almost always metal cations, the guests are solvent or gas molecules absorbed or trapped in the pores or otherwise ions that balance the charge of the framework. Linkers and connectors define versatile structures characterized by very flexible dimensionality (1D chains, 2D layers or 3D frameworks) and by charge "diversity". Figure 1 gives a schematic representation of a typical framework structure with all the building block types.

One of the main issues in this research field is the design of materials having specific structural and functional features. This task implies, on one hand, the prediction of solid state structures, which is nowadays a huge branch of science [3]. On the other hand, understanding the origin of a given property is fundamental because it allows engineering new functional compounds, reducing the efforts of experimental screening. Therefore, the research in this direction necessarily involves studies on the electronic structure, that determines the molecular and supramolecular geometries as well as many properties (thermal, electric, electronic, magnetic, optic etc.).

Modeling can be done at different levels, ranging from full quantum mechanical to semi-empirical methods based on parameterized descriptors and reducing the problem to semi-classical physics.

The electron distribution of a material plays a very important role because it represents a bridge between physics and chemistry. In fact, the laws of quantum mechanics predict the average 
distribution and flow of the electrons, respectively the position electron density $\rho(\mathbf{r})$ and the current density $J(\mathbf{r})$, two quantities that allow chemists to understand the functions and the evolution of compounds [4].

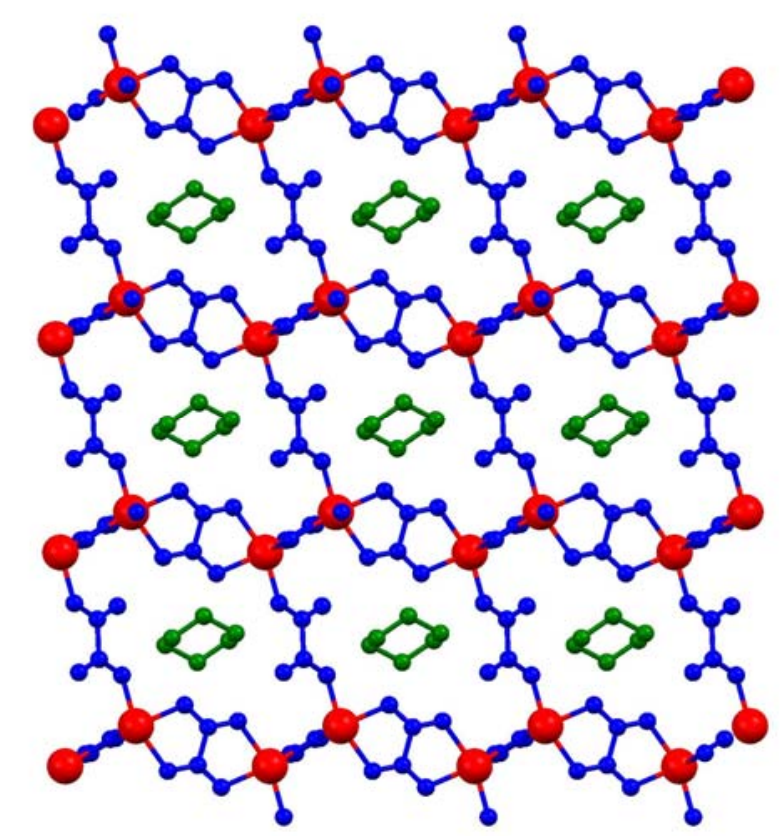

Figure 1. A typical metal organic frameworks, characterized by metal connectors (red, larger balls), the organic linkers (blue molecules bridging the metals) and the guests (green molecules in the cavities). This particular structure is $\mathrm{Zn}_{2}\left(\mathrm{C}_{2} \mathrm{O}_{4}\right)_{3}\left[\mathrm{C}_{4} \mathrm{H}_{12} \mathrm{~N}_{2}\right]$, calculated from ab initio periodic Density Functional Theory, based on the experimental structure reported in ref [5]. The connector is $\mathrm{Zn}^{2+}$, the linker is oxalate (with two different coordination modes) and the counter-ion guest is piperazinium.

The importance of electron density resides in its inherent, but hidden, correlation with energy [6] and the fact that it is an observable, at variance from the wave function. Therefore, not only $\rho(\mathbf{r})$ can be calculated from first principle quantum mechanics, but it can also be measured experimentally. The most adopted technique is the diffraction of X-rays from crystals, because the elastic X-ray scattering of a periodic structure is the Fourier transformation of its electron density [7].

From the electron distribution, many features of a material become available. For example, the way in which the molecular bricks are held together can be revealed by an accurate analysis of $\rho(\mathbf{r})$. This is possible by studying the electric forces acting between molecules, under the assumption that only electrostatics is active, or by a quantum theory of atoms in molecules (QTAIM) [8] analysis, necessary if electron exchange is taking place between molecules and therefore a semi-classical reductionism cannot be straightforwardly adopted.

Although the word material does not necessarily indicate compounds in the solid state, in practice this word is often associated with solids, either amorphous or crystalline. Moreover, given the enormous analytical potential of scattering techniques from periodic objects and the higher control of the 
functionalities in three-dimensional ordered systems, scientists are often focused on crystalline materials. It is not surprising, in fact, that the crystal engineering has vigorously emerged in the last two decades as a new branch of science, reaching now a quite mature status.

We revise in this commentary the state of the art on investigation of the binding between linkers and connectors in metal organic frameworks, as well as the intermolecular interactions occurring between the guests and the framework. We will describe a general approach for modeling the electron density of metal organic materials, based on a simplified and easily exported picture of these materials, to be used for the subsequent studies of their properties.

\section{Modeling}

Quantum mechanics is of course the most accurate and reliable theory to study the electronic structures of materials. However, the size of the problem may challenge the current computational resources, especially for metal organic frameworks, forcing the adoption of some approximations. Therefore, accurate but simpler and computationally less expensive descriptors are extremely useful. In analogy with other branches of science, for example drug design, electron density based descriptors are potential candidates to allow reliable characterization and simulations of materials. There are two reasons: 1) electron density partitioning is very practical, can be done in real space at relatively low costs and it can guarantee exportability; 2) electron density is itself a quantum mechanical observable, therefore it reflects, at least in part, the action of non classical forces on electrons.

As mentioned above, X-Ray diffraction is still the elective technique for the experimental determination of the electron density distribution, although other methods are becoming popular, in combination with [9] or in alternative to [10] elastic scattering of X-rays. Many progresses occurred since the first observations of aspherical electron distributions of atoms in small molecular crystals, described by the so-called deformation densities [11]. Nowadays, $\rho(\mathbf{r})$ is more easily obtained also in very large molecules, even containing heavier elements for which the more polarizable valence electrons are in defect compared to the almost imperturbable core electrons [12]. Among the possible models proposed in the literature, the multipolar expansion is by far the most adopted one. According with Stewart [13], the total electron density could be projected onto atom-centered electron density functions (pseudoatoms):

$\rho(\mathbf{r})=\sum_{i=1}^{n a t} \rho_{i}(\mathbf{r})$

Within the Hansen-Coppens formalism [14], the electron density of each pseudoatom is then expanded in three major components: the core density (typically a rigid and spherical term with frozen population), a spherical valence density (with variable population and a contraction/expansion flexibility) and a deformation valence density (with variable multipole coefficients and expansion/contraction flexibility): 


$$
\rho_{i}(\mathbf{r})=P_{i, \text { core }} \rho_{i, \text { core }}(\mathbf{r})+P_{i, \text { valence }} \kappa_{i}^{3} \rho_{i, \text { valence }}(\mathbf{r})+\sum_{l=0, l_{\max }}\left[\kappa_{i}^{\prime 3} R_{l}\left(\kappa_{l}^{\prime} \mathbf{r}\right) \sum_{m=0, l} P_{l m \pm} y_{l m \pm}(\mathbf{r} / r)\right]
$$

The parameters $P_{l m \pm}, P_{\text {valence, }} \kappa$ and $\kappa^{\prime}$ are typically refined within a least square procedure, together with positional and thermal parameters, as it occurs in a normal crystal structure refinement. $y_{l m \pm}$ are spherical harmonic functions. $\rho_{\text {core }}, \rho_{\text {valence }}$ and $R_{l}$ are radial density functions, normally taken from energy optimized atomic wave functions for isolated atoms [15]. The variables $\kappa$ and $\kappa^{\prime}$ allow the radial flexibility, whereas the spherical harmonics functions guarantee the aspherical flexibility. A more detailed treatment of the core shells has been recently proposed [16]:

$\rho_{i}(\mathbf{r})=\sum_{n=1, \text { electron-shell }}\left\{P_{i n} \kappa_{i n}^{3} \rho_{\text {in }}(\mathbf{r})+\sum_{l=0, l_{\max }} \kappa_{\text {inl }}^{\prime 3} R_{\text {inl }}\left(\kappa_{\text {inl }}^{\prime} \mathbf{r}\right)\left[\sum_{m=0, l} P_{i n l m \pm} y_{l m \pm}(\mathbf{r} / r)\right]\right\}$

Here each electronic shell is independently refined, which is of course increasing enormously the number of variables.

Noteworthy, pseudoatomic projection could be applied in direct space (i.e. in the real space $\mathfrak{R}^{3}$ of the electron density) or in a transformed space (for example, the Fourier transformed space $\mathfrak{I}$ of the scattering vectors). The latter is the way to refine a model against the measured X-ray intensities ${ }^{1}$ at Bragg positions. In fact, the X-ray structure factor $\mathbf{F}$ for a given scattering vector $\mathbf{H}$ is given by:

$$
\mathbf{F}(\mathbf{H})=\int_{V} \bar{\rho}(\mathbf{r}) e^{2 \pi i \mathbf{H} \bullet \mathbf{r}} d \mathbf{r}
$$

where $\bar{\rho}(\mathbf{r})$ is the thermally averaged electron density distribution (i.e. the correlation between the electron density and the Temperature dependent nuclear motion). In keeping with the atomic expansion, $\mathbf{F}(\mathbf{H})$ can be approximated as:

$$
\mathbf{F}(\mathbf{H})=\sum_{i} f_{i}(\mathbf{H}) T_{i}(\mathbf{H}) e^{2 \pi i \mathbf{H} \circ \mathbf{r}}
$$

where $f_{i}$ is the atomic scattering factor and $T_{i}$ is the atomic Debye-Waller factor for the thermal motion (also called temperature factor). The multipolar expansion implies a breakdown of $f_{i}$ into different contributions:

$$
f_{i}(\mathbf{H})=P_{i, \text { core }} f_{i, \text { core }}(\mathbf{H})+P_{i, \text { valence }} f_{i, \text { valence }}(\mathbf{H})+\sum_{l=0, l_{\max }} P_{i, l m \pm} f_{i, l m \pm}(\mathbf{H})
$$

where the $f_{i}^{\prime}$ s are the Fourier transformation of the radial and angular terms of equation (2).

One of the most important advantages of the Hansen-Coppens formalism is the definition of a local coordinate system for each pseudoatom. In fact, while in most crystallographic applications there is no need to describe an atom in a reference system different from that of the unit cell, internal molecular coordinates are instead necessary especially for the "transferability" of electron density parameters

\footnotetext{
${ }^{1}$ Or any other radiation that brings electron density information, for example electrons.
} 
between chemically equivalent atoms. A given functional group shows a typical electron density distribution in all molecules in which it is contained. This similarity is however evident in the multipolar description only with respect to an internal coordinates system, which is rarely coincident with the unit cell basis, given that molecules normally crystallize with much lower symmetry than their point group.

This modeling choice has, indirectly, anticipated the work which is characterizing most of the modern applications of charge density analysis: the exportability of multipolar parameters [17] from one atom of a given functional group to a chemically equivalent atom in a more complex system. This approach in fact requires that an individual functional group or an atom is defined with respect to a local coordinate system, which guarantees the exportability of the parameters and, in addition, it allows defining restraints or constraints to chemically equivalent atoms that are not crystallographically equivalent.

So far, multipolar databases were used to study molecular crystals of biological interest $[18,19]$. The various approaches have dealt with the problem of correct classifications of atoms, based on chemical types. The invariant-atom (invariom) method is the most generalized [20], as it classifies atoms based on pure topological criteria. Other database methods, like ELMAM [21] and the UBDB [22] libraries, classify atoms based on more traditional chemical criteria. Interestingly, the databases could be created from experimental observations (i.e. from refinements of a number of proto-type molecular crystals, whose X-ray diffraction was accurately measured [17a]) or from theoretical calculations with fitting in direct [23] or reciprocal space [17b]. The hypothesis of transferable atoms in biomolecules was strongly supported also by Matta and Bader [24] using electron density partitioning, but without a parameterization in terms of atom projected multipoles.

The studies that appeared in the literature so far had two main objectives: a) the accurate models of atomic scattering factors from databases of atomic multipoles allowed to improve the quality of structural refinements of medium or low resolution data collections on macromolecules, or even proteins [25]; b) the accurate electron density reconstructed from the databases enabled the low cost evaluation of properties of these molecules in crystalline environment, in particular electrostatic moments and interaction energies. A very recent publication compared the performances of the various approaches both in terms of refinement improvements and evaluation of electrostatic properties [26]. The inherent difficulty of the electron density breakdown into transferable multipoles for macromolecules is that the cut necessarily occurs at a covalent bond (for example a peptidic bond). The main implication is that total electron population may not be constant and requires renormalization. Nevertheless, the results in this direction are promising and more progresses are expected in due course. 
Although the field of metal organic materials could be somewhat similar to that of macromolecules, similar kinds of models have not been proposed so far. Some electron density studies on MOFs have been reported [27], mainly focused on the analyses of chemical bonding features of these materials. Spackman [28] proposed an Hirshfeld's partitioning [29] to define molecular or fragments' volumes within a crystal. The Hirshfeld surface defines a volume containing at least $50 \%$ of the electron density of the molecule or fragment based on the simple summation of the electron densities of each atom in isolation. Although this method is not quantum mechanical, it became quite a popular tool for crystal engineering analysis, especially to characterize the binding properties of a molecule and the area of influence of a given intermolecular interaction [28b].

Correlation between electron density and material properties is more rarely carried out, especially in metal organic materials. This requires not only details of local bonds but also evaluation of non-local interactions and global propagation of the property.

We summarize here the first application of an electron density breakdown into building blocks of molecular materials, a valid tool for rationalization of a given material property and careful analysis of its source in correlation with the crystal structure. The method is intentionally preserving the simplicity of the pseudoatom expansions. The total electron density is the sum of the building blocks densities:

$$
\rho(\mathbf{r})=\sum_{i=1}^{N_{B B}} \rho_{i, B B}(\mathbf{r})
$$

where $N_{B B}$ is the number of building blocks and $\rho_{i, B B}(\mathbf{r})$ is the electron density of a building block, obtained, for example, from a quantum mechanical calculation even at a high level of theory, given that in general it would not be so expensive. For the metal connectors, the BB density coincides with the electron density of the cation in isolation. Because building blocks are, normally, closed-shell molecules or ions, this approximation avoids breaking electron paired chemical bonds. It should not escape, however, that this approximation is implying no covalency between the linker and the connector, which is of course not true in general. In fact, the amount of shared character in metalligand bonds is in general quite significant. However, it was noted already by Coppens et al. [30], that the overlap between metal and ligand orbitals is sufficiently small that the d-orbital electron population of a metal can be reconstructed form the coefficients of a multipolar expansion. This is possible only assuming no mixing of the metal orbitals through overlap with the ligand orbitals. Despite approximated, this assumption has always produced orbital populations from experiments that could match with sufficient accuracy those calculated from ab initio wave functions, where the mixing is implicitly assumed.

For sake of simplicity, the building block density is described in terms of atom expanded multipoles, fitted in the reciprocal space $\mathfrak{I}$ (although this approach could also take advantage from the recent progresses in direct space fitting): 


$$
\rho_{i, B B}(\mathbf{r})=\sum_{j=1}^{\text {Natom }(i)} \rho_{j}(\mathbf{r})
$$

The atomic densities $\rho_{j}(\mathbf{r})$ are then expanded as in equations (2).

The building block model maintains the exportability of the other databases approaches, using of course local coordinate systems which are easily defined. A database can be easily constructed by storing all symmetry independent atoms of a given building block, calculated from ab initio molecular orbital wave functions. A typical level of theory could be the Density Functional Theory, using an hybrid functional, which are normally returning more accurate description of the electron density distributions.

In a recent work [31], we have examined the application of this method to an important class of compounds, like the $\mathrm{Zn}$ oxalate frameworks. The choice of $\mathrm{Zn}^{2+}$ cation is justified because this is a closed shell cation with a formal full occupancy of the $3 \mathrm{~d}$ shell. Accurate calculations and measurements, however, showed that the cation is anyway slightly distorted from perfect sphericity [27a]. Within the QTAIM partitioning, one could see the effects of the partial covalency in the electron delocalization indexes [32], a measure of the amount of electron pairs shared by two atoms, which significantly deviates from 0 for all the Zn-O bonds. This implied some improvements to the model, like using densities of the cation calculated in a field of negative charges surrounding the metal with a given stereochemistry (thus distinguishing for example tetrahedral or octahedral coordination). Further improvement could be corrections of linkers density, including perturbation due to the field of the cations. This could be important in particular for anionic linkers, which are of course more polarizable. For example, the distributed atomic polarizabilities [33] are shown in Figure 2 for oxalic acid and the oxalate anion. Although having the same number of electrons, the isotropic polarizability of the anion is ca. $60 \%$ larger. The atomic polarizability tensors are in fact much more prolated. As a consequence, the anionic linker will be much polarized when coordinated to a cationic connector.

The subtle problems connected with proper modelling of the building blocks are exemplified by the almost identical quality of the fit of a simple metal organic material like $\mathrm{Zn}\left(\mathrm{C}_{2} \mathrm{O}_{4}\right)\left(\mathrm{H}_{2} \mathrm{O}\right)_{2}$, characterized by infinite chains of Zn-oxalates, with two water molecules coordinated at the apexes of the $\mathrm{Zn}$ octahedrons (see Figure). In fact, an hypothetical "neutral" model, i.e. refined preserving the electroneutrality of the building blocks, would fit the theoretical or experimental structure factors in quite a similar way as a chemically sensible charged-constrained refinement, i.e. preserving the formal charge of the linker and the connector $\left(\mathrm{R}_{1}=0.0053\right.$ vs 0.0052 , for neutral or charged model refinement, respectively $)^{2}$. The reason for this similarity is due to the diffuse nature of the anionic

\footnotetext{
${ }^{2} \mathrm{R}_{1}=\Sigma|| F_{\mathrm{o}}|-| F_{\mathrm{c}}|| \Sigma\left|F_{\mathrm{o}}\right|$ where $F_{o}$ is the observed structure factor modulus and $F_{c}$ the structure factor calculated with a given model.
} 
electron cloud around the linker, easily fitted also by the external electronic shell of the metal $(4 \mathrm{~s}$ orbital).
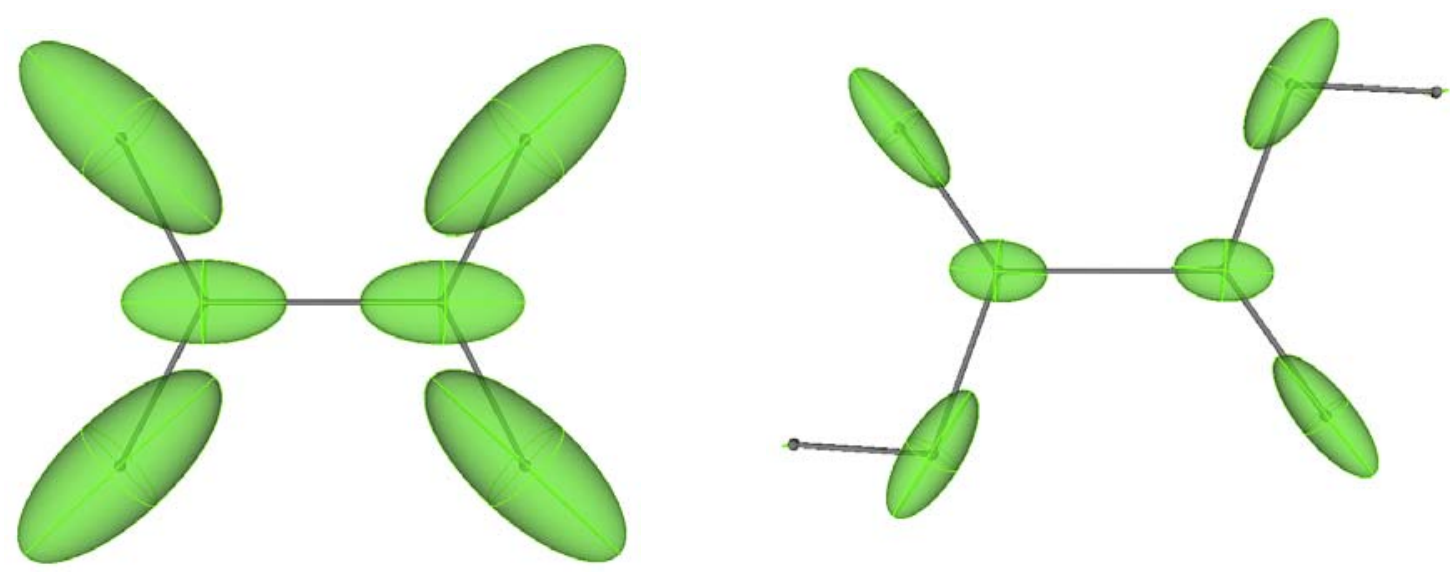

Figure 2. The distributed atomic polarizabilities in oxalate (left) and oxalic acid, calculated with Density Functional Theory (at B3LYP/6-311++G(2d,2p) level of theory). The atomic ellipsoids (measured in $\AA^{3}$ ) are plotted assuming a $0.3 \AA$ : $1.0 \AA^{3}$ scale. The plot shows the larger polarizability of the anion.

Figure 3, illustrate the differences between the two models, showing the total electron density distribution and the electrostatic potential in a typical section of a Zn-oxalate honeycomb layer (a very common packing motif). If using a space partitioning of the electron density, based on the topological criteria of the QTAIM, then the saddle points between the Oxygens of the oxalate and the Zn caiton are the boundaries of linkers and connectors, resulting in a positively charged connector $(\mathrm{ca} .+1.4)$ and a negatively charged linker. On the other hand, if we use as boundaries the saddle points of the electrostatic potential $\varphi(\mathbf{r})[34]$

$\varphi(\mathbf{r})=\int \frac{\rho\left(\mathbf{r}^{\prime}\right)}{\left|\mathbf{r}-\mathbf{r}^{\prime}\right|} d \mathbf{r}^{\prime}$

then, because of the Gauss theorem, the volumes defined within these surfaces are necessarily neutral. Thus the distinction between a chemically sensible charged model and a neutral model (mathematically compatible but less useful for a chemical interpretation) is a shift of the building block boundaries. Of course, a model in agreement with chemical intuition would be preferable for applicative purposes and it is therefore preferred, also because it would preserve the easier calculation of building blocks in the form of closed shell molecules or ions. The gap between the formal \pm 2 charge and the actual \pm 1.4 gives an idea of the approximation of the method and the degree of basis set superposition error. 


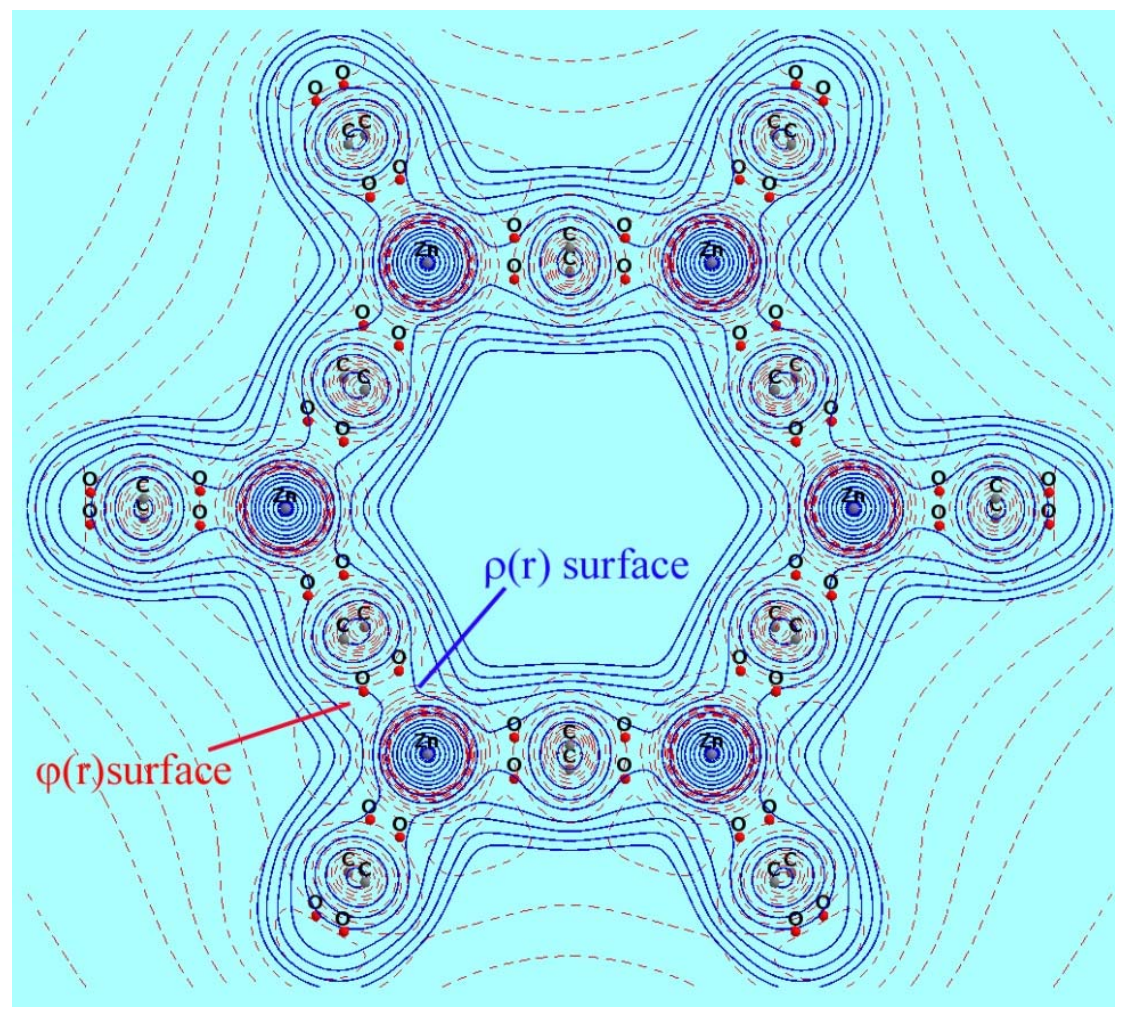

Figure 3: The electron density (blue) and negative electrostatic potential (red, dashed) contours of a $\left[\mathrm{Zn}_{6}\left(\mathrm{C}_{2} \mathrm{O}_{4}\right)_{12}\right]^{12-}$ unit, a fragment of a typical honeycomb layer of $\mathrm{Zn}$-oxalates. Atoms are depicted as small spheres. The saddle between the $\mathrm{Zn}$ connector and the oxalate linker indicate the occurrence of a surface separating the two building blocks. If the electrostatic potential is used for this separation, the surface define a larger volume for $\mathrm{Zn}$ and smaller for the oxalate (as the building blocks must be neutral in this case). The electron density partitioning, instead, produce \pm 1.4 electron charges.

As discussed above, the building block is a rather simplified description of the charge density in a metal organic material, which has some undoubted advantages. In fact, apart for the very easy description, the exportability of the density, it is important to stress that calculations of extended solids could be reduced to simple additive terms, thus minimizing computational costs of functions and properties form this electron density distribution.

Simplified models are necessary for predicting the structural features and/or the chemical and physical properties of a material. The recent trends of theoretical approaches to material design are in fact oriented towards the optimization of the properties. This means searching for compounds that fulfil specific structural requirements and guarantee optimized properties [35].

While the first principle treatment of inorganic materials is favoured by the smaller size of these systems, molecular organic or organometallic materials are definitely more complex. For this reason, simplified models for the molecular building blocks are extremely useful. 


\section{Forces between Building Blocks}

In order to predict structures and properties of a molecular material it is essential to evaluate the forces active between the building blocks. As anticipated above, MOFs are often characterized by a large charge flexibility which means that the role of the electrostatic interactions could be very important, but also quite tunable.

The interaction between closed-shell units is in general partitioned in:

a) The electrostatic interaction: it can be calculated exactly from $\rho_{i, B B}(\mathbf{r})$. The corresponding energy is given by:

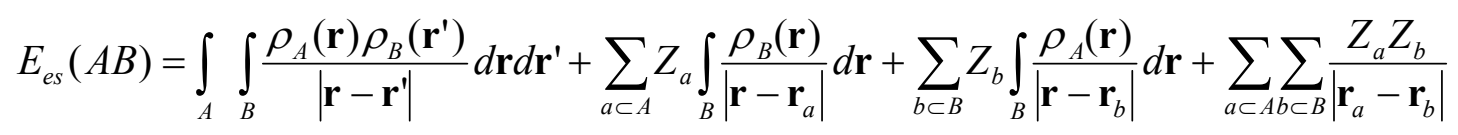

At large distances, it can be simplified in atom expanded multipole moments, thanks to Buckingham formalism [36]:

$$
E_{e s}(A B)=\sum_{a \subset A b \subset B} T q_{a} T q_{b}+T_{\alpha}\left(q_{a} \mu_{\alpha}-q_{b} \mu_{\beta}\right)+T_{\alpha \beta}\left(\frac{1}{3} q_{a} \Theta_{\alpha \beta, b}+\frac{1}{3} q_{b} \Theta_{\alpha \beta, a}-\mu_{\alpha, a} \mu_{\beta, b}-\mu_{\alpha, b} \mu_{\beta, a}\right)
$$

with implicit summations (Einstein convention) over $\alpha, \beta \ldots$ directions, $a$ and $b$ indexes run over all atoms belonging to $A$ or $B$ building blocks, $\mu_{\alpha}$ and $\Theta_{\alpha \beta}$ are electric dipole and quadrupole moment components, whereas $T_{\alpha \beta \gamma \ldots}$ are the symmetrical interaction tensors $\left(\nabla_{\alpha} \nabla_{\beta} \nabla_{\gamma} \ldots\left(\mathbf{r}_{\mathrm{ab}}\right)^{-1}, \mathbf{r}_{\mathrm{ab}}\right.$ being the vector from the origin of a to $b$ ). The sum extends up to the higher multipole-multipole interaction and it generally converges for $l_{a}+l_{b}>5$ ( $l$ being the order of the multipolar expansion on center $a$ and $b$, respectively).

b) The induced polarization: depending on its electric dipole (hyper)polarizabilities, a building block is polarized by the field of the other BBs. This produces a permanent change to the electron distribution and therefore an additional electrostatic energy. The calculation of this term requires some approximation, because calculating the polarizability and the electric field at each point in space would be quite expensive. Therefore, one can calculate the average electric field produced by the Building Block $B$ at the position of each atomic position of Building Block $A$ and calculate the induction from

$$
E_{\text {ind }}(A B)=\sum_{j \subset A} \frac{1}{2} \boldsymbol{\alpha}_{A, j} \mathbf{E}_{B}^{2}\left(\mathbf{r}_{j}\right)+\sum_{j \subset B} \frac{1}{2} \boldsymbol{\alpha}_{B, j} \mathbf{E}_{A}^{2}\left(\mathbf{r}_{j}\right)
$$

where $\mathbf{E}(\mathbf{r})$ 's are the electric fields produced by the building blocks and $\alpha_{j}^{\prime}$ s are the atomic polarizabilities.

c) The exchange-repulsion: this is the most common name given to a local destabilization experienced by electrons, obeying to Fermi-Dirac statistics for Fermion particles that cannot occupy the same position in space, with the same spin. The energy of this interaction can be approximated with a potential, for example in the form of $\mathrm{ar}^{-12}$ function [39]: 
$E_{r e p}(A B)=\sum_{a \in A} \sum_{b \in B} \frac{\sigma_{a b}}{r_{a b}^{12}}$

or an exponential function

$E_{\text {rep }}(A B)=b_{a} b_{b} \exp \left[\left(c_{a}+c_{b}\right) r\right]$

These formula runs over each atom of the building blocks and the coefficients are taken from fittings to potentials derived from an electron gas model for each atom pair [37] or other more empirical formulations [38].

d) London forces: the non permanent induced polarizations produce a weaker extra stabilization (dispersion) between molecules. A precise calculation of these forces and the corresponding energies would require configuration interaction calculations, which are quite prohibitive. However, approximate force fields, like Lennard-Jones potentials [39], are able to reproduce the dispersive interaction between two building blocks $\mathrm{A}$ and $\mathrm{B}$.

$E_{\text {disp }}(A B)=\sum_{a \in A} \sum_{b \in B} \frac{C_{a b}^{(6)}}{r_{a b}^{6}}+\frac{C_{a b}^{(8)}}{r_{a b}^{8}}+\frac{C_{a b}^{(10)}}{r_{a b}^{10}}+\ldots$

Again the summation runs over all atoms of each building blocks and the coefficients are obtained from fitting the potential against calculated atomic polarizabilities [37]. Following the original formulation by London [40], in fact, the dispersion energy could be calculated from the isotropic dipolar polarizabilities $\alpha$ and ionization energies of the two building blocks:

$E_{\text {disp }}(A B)=-\frac{3}{2} \frac{E_{A}^{i o n} E_{B}^{i o n} \alpha_{A} \alpha_{B}}{\left(E_{A}^{i o n}+E_{B}^{i o n}\right) r_{A B}^{6}}$

or otherwise as a summation of atom-atom pairwise interactions, if using distributed atomic polarizabilities [33]:

$E_{d i s p}(A B)=-\frac{3}{2} \sum_{a \subset A} \sum_{b \subset B} \frac{E_{A}^{i o n} E_{B}^{i o n} \alpha_{a} \alpha_{b}}{\left(E_{a}^{i o n}+E_{b}^{i o n}\right) r_{a b}^{6}}$

e) electrophilic-nucleophilic interactions (Hydrogen Bonding): in case of incipient covalent mechanisms between the building block, for example Hydrogen Bonding or more generally donoracceptor interactions [41], all the above approximations may require further corrections, typically based on some empirical correlation with the distance between the interacting atoms [42].

The sum of all the terms a)-e) is the interaction energy $E_{\text {int }}$ between building blocks, which is useful to evaluate some properties of a material, for example accessible sites for guest molecules. 


\section{Properties}

As anticipated in the introduction, materials are researched for their ability to react upon some stimulus. These responses attribute a mono- or poly-functional behavior: some of them are directly calculated from the electron density distribution, for example the electric polarization; others comes from changes of the electron density upon some perturbation, like the electric susceptibilities; many transport properties, for example electric or thermal conductivity, are not directly correlated with the space averaged electron density distribution, although it is often observed that features of the chemical bonding could be fingerprints of these properties [43].

The building block interactions summarized in the previous section can be useful to predict some properties of the porous metal organic compounds. For example, the ion exchange mainly depends on the interaction energies between the ionic guests and the frameworks, which could accelerate or decelerate the diffusion processes. More in general, the capacity of the framework to trap, select and perhaps react with some guests depends on the forces acting between the building blocks. With this concept in mind, we have developed an interaction energy mapping: a guest is used to sample the rigid framework in order to locate sites more adapted for binding. The function $\mathrm{E}_{\text {int }}(\mathbf{r} ; \alpha, \beta, \gamma)$ is calculated as $E_{\text {int }}(\mathbf{r} ; \alpha, \beta, \gamma)=E_{e s}(\mathbf{r} ; \alpha, \beta, \gamma)+E_{\text {ind }}(\mathbf{r} ; \alpha, \beta, \gamma)+E_{\text {disp }}(\mathbf{r} ; \alpha, \beta, \gamma)+E_{\text {ex-rep }}(\mathbf{r} ; \alpha, \beta, \gamma)$

where $\mathbf{r}$ is the position vector of the guest center of mass and $(\alpha, \beta, \gamma)$ are the Eulerian angles of the main inertial axis with respect to the framework coordinate system. $E_{\mathrm{es}}, \mathrm{E}_{\mathrm{disp}}, \mathrm{E}_{\text {ind, }} \mathrm{E}_{\mathrm{ex}-\mathrm{rep}}$, are the relative interaction energies calculated between the guest at coordinates $(\mathbf{r}, \alpha, \beta, \gamma)$ and the rigid $3 \mathrm{D}$ framework or $2 \mathrm{D}$ layer structure. At each $\mathbf{r}$, the guest is rigidly rotated until the $(\alpha, \beta, \gamma)$ coordinates are found which minimize the interaction energy. To reduce the computational costs, the following approximations are made: a) at large separation (above $10 \AA$ ) between a building block of the framework and the sampling guest, the total multipole moments of the building blocks are used to evaluate $\mathrm{E}_{\mathrm{es}}$ via Buckingham approximation (11); b) for intermediate distances, the distributed atomic multipole moments are used in the Buckingham summation; c) for atom-atom distances within van der Waals radii, $\mathrm{E}_{\mathrm{es}}$ is evaluated more accurately using the exact potential approach suggested by Volkov et al. [44], thus solving exactly equation (10).

This procedure enables testing the hosting ability of a porous material. For example, the Solvent Accessible Volume (SAV), a quantity often used in crystal engineering, can be computed with higher accuracy. SAV is normally evaluated simulating atoms with hard spheres, thus neglecting the actual interactions between them. SAV can be instead determined using the interaction energy as a threshold to locate available sites (characterized by stabilizing energy) and unavailable sites (characterized by destabilizing energy). It is interesting that also this kind of approach is imported in material design from computational studies on proteins [45]. In addition, this interaction energy mapping could be useful to solve problems connected with disorder of guest molecules in channels or cavities of the host frameworks [46]. 
One example of this method is shown in Figure 4. A typical honeycomb charged layer of $\mathrm{Zn}$-oxalate is sampled with a mono-protonated piperazinium cation, which is one of the extra-framework cation in some of these materials [5]. The comparison with the electrostatic potential map of the same framework is very instructive. In fact, $\varphi(\mathbf{r})$ is also a sampling of the framework structure, though using a point charge, which therefore does not reveal the anisotropies of the guest (but only those of the host) and - more importantly- it neglects the energies produced by the other forces. As a result, the electrostatic potential is highly negative (i.e. stabilizing for a positive charge) close to the oxalate Oxygens that bear the most negative charge, but only weakly stabilizing at the center of the honeycomb hole. The $\mathrm{E}_{\text {int }}(\mathbf{r})$ map, instead, shows the more stabilizing region at the center of the pore, a result coming from the balance between electrostatic attraction and short range repulsion. This is in fact one of the sites found experimentally for the molecular extra-framework cations in these kind of structures, see Figure 4. Another region suitable for the cations is that in between the layers (not shown in the figure).
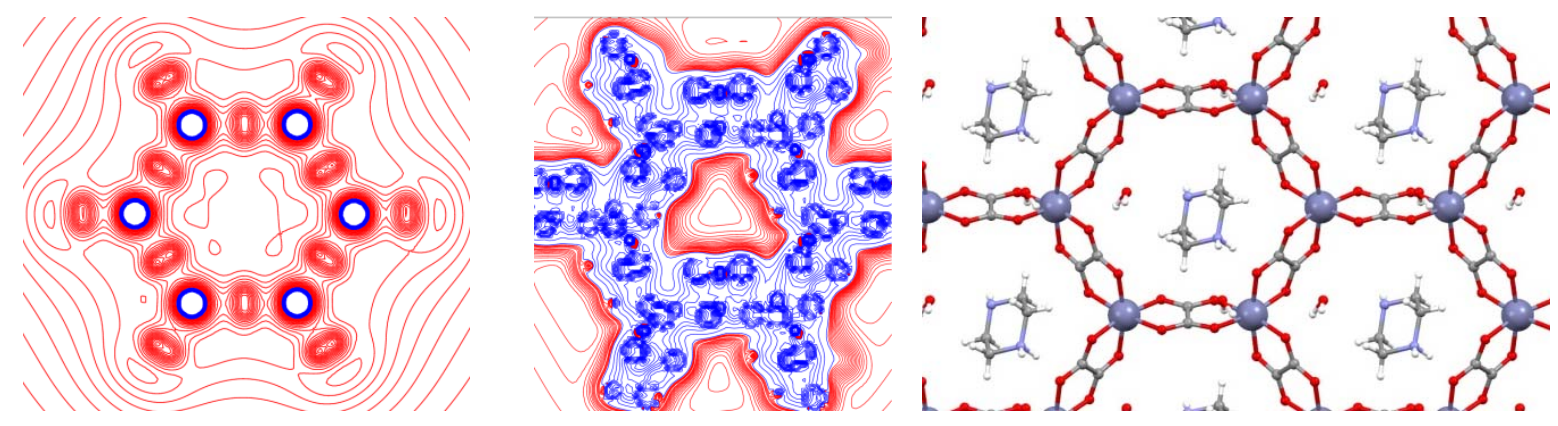

Figure 4. Left: electrostatic potential (red contour negative potential; blue contours positive potential) in a model honeycomb layer of $\mathrm{Zn}$ oxalate; center: the interaction energy map of the honeycomb pore sampled by a mono-protonated piperazine (red contours stabilizing energy; blue contours destabilizing energy); right: the structure of $\mathrm{C}_{14} \mathrm{H}_{32} \mathrm{~N}_{4} \mathrm{O}_{16} \mathrm{Zn}_{2}$ [5] a layered structure with water molecules, monoprotonated piperazinium cations in the honeycomb holes and dications (not shown in the picture) in between the layers. Electrostatic potetnial and inetraction energy maps have been caclulated with a modified version of XD2006 [47]

Another application of the building block approach is using their (hyper)polarizabilties to predict, at least approximately, the optical properties of crystal species. The first polarizability $\boldsymbol{\alpha}_{B B}$ of a building block can be accurately calculated using ab initio quantum mechanics. As shown in Figure 2, a partitioning could be applied, based on the electron density, so that the atom-distributed polarizabilities $\alpha_{i}$ can be obtained [33]. In this way,

$\boldsymbol{\alpha}_{B B}=\sum_{i=1}^{N a t} \boldsymbol{\alpha}_{i}$ 
The additivity of the atomic polarizability is guaranteed if the electron density partitioning is exact (i.e. it reconstructs the total density) as for example QTAIM. The atomic partitioning is particularly important because it allows corrections for the perturbation effects due to other building blocks. For example, the polarizability of a building block within a secondary building unit $(\mathrm{SBU})^{3}$ can be reconstructed by summation of the polarizabilities of all atoms belonging to that building block, but calculated in the entire SBU.

The optical properties of a crystal depend on the susceptibilities that are correlated with the building block polarizabilities. For example, the linear susceptibility is given by

$$
\chi=\frac{4 \pi}{V} \sum_{j=1}^{N_{B B}} \boldsymbol{\alpha}_{j}
$$

where $V$ is the total volume of the unit cell and $N_{B B}$ is the number of building blocks in the unit cell. $\boldsymbol{\alpha}_{B B}$ 's are of course transformed from the coordinate system of the building block into the crystal coordinate system. A correction is necessary, however, in order to include the long range effects of a periodic distribution of polarizable building blocks, as suggested by Dunmur [48] and adopted by Spackman et al. [49]. This means that each BB polarizability should be corrected for the apparent enhancement, caused by the electric field of the induced dipole moments of all other building blocks in the crystal. This effect is explained by Dunmur [48] using classical electrostatics. Of course, if the building block polarizability was first calculated within a SBU, the effect of the first coordination sphere is already included at quantum mechanical level, thus the induced electric field perturbation should be computed including induced dipoles from the second coordination sphere on. This approach was used for evaluation and rationalization of linear optical properties in simple oxalate salts of amino acids [50], but could be adopted also for more interesting metal organic materials. The importance of this kind of studies is evident, if we consider that from linear or nonlinear susceptibilities all optical and electro-optical effects are derived.

\section{Conclusion}

We have shown in this brief commentary some potential applications of the electron density analysis of metal organic materials, in particular using the building block breakdown.

Electron density partitioning provides suitable descriptors that could be easily exported from one species to another. Of course, there are two main requirements: a) the descriptors should accurately reconstruct the total electron density and the related properties; b) the computational costs of this treatment should be significantly smaller than those of a full quantum mechanical treatment of the entire system (which is of course inherently more accurate). For both points, distributed atomic multipoles or polarziabilties appear very adequate, because they can easily reproduce the major

\footnotetext{
${ }^{3} \mathrm{~A}$ unit composed of by the first coordination of building blocks around a given connector or linker.
} 
features of the building block electron density distribution or polarizability including perturbations caused by their assembly and, at the same time, they allow quite simplified and rapid calculations of the crystal properties.

We envisage the usage of building the block approach to evaluate also mechanical properties of crystals, for example elastic constants, easily available from multipole moments [51]. Moreover, the highly reduced computational costs could stimulate more sophisticated molecular dynamic calculations that could enable evaluation of transport properties and adsorption properties.

Within static mechanic calculations, the building block approach could be also adopted to extend the material design through property optimization [35], which means maximization or minimization of a given material property using structural and chemical variables.

\section{Acknowledgements}

We thank the Swiss National Science Foundation, project Nr. 141271 for financial Support.

\section{References}

[1] Tranchemontagne D J, Mendoza-Cortés J L, O’Keeffe M and Yaghi O M 2009 Chem. Soc. Rev. 381257.

[2] Functional hybrid materials (Wiley-VCH, Berlin 2004) ed P Gomez-Romero and C Sanchez.

[3] Modern Methods of Crystal Structure Prediction (Wiley-VCH, Berlin 2010) ed A R Oganov.

[4] Bader R F W Enciclopedia of Computational Chemistry. 2002. DOI: 10.1002/0470845015.caa012.

[5] Vaidhyanathan R, Natarajan S and Rao C N R 2001 J. Chem. Soc. Dalton Trans. 699.

[6] Hohenberg P and Kohn W 1964 Phys. Rev. B 136864.

[7] a) Coppens P. X-ray Charge Densities and Chemical Bonding (Oxford University Press: Oxford, 1997); b) Koritsanszky T and Coppens P. 2001 Chem Rev. 1011583.

[8] Bader R F W Atoms in Molecules: A Quantum Theory. (Oxford Science Publications, Oxford 1990).

[9] Gillet J M and Koritsanszky T Past, Present and Future of Charge Density and Density Matrix Refinements Modern charge density analysis (Springer 2012) ed C Gatti and P Macchi.

[10] Nakashima P N H, Smith A E, Etheridge J and Muddle B C 2011 Science 3311583.

[11] Coppens P 1967 Science 158 1577. A deformation density is the difference between the total electron density and the superposition of spherical, unperturbed atomic densities. It can be static or dynamic, depending whether thermal smearing is included or not; it can be experimental or theoretical (the reference pro-molecule being always theoretical, of course).

[12] Stevens E D and Coppens P 1976 Acta Cryst. A32 915.

[13] Stewart R F 1969 J. Chem. Phys. 514569.

[14] Hansen N K and Coppens P 1978 Acta Cryst. A34 909.

[15] a) Clementi E and Roetti C Tables of Roothaan-Hartree-Fock Wavefunctions, Special Issue in Atomic Data and Nuclear Data Table (Academic Press, New York, 1974); b) Clementi E and Raimondi D L 1963 J. Chem. Phys. 382686.

[16] Fischer A, Tiana D, Scherer W, Batke K, Eickerling G, Svendsen H, Bindus N and Iversen B B $2011 \mathrm{~J}$. Phys. Chem. A. 11513061.

[17] a) Pichon Pesme V, Lecomte C and Lachekar H. 1995 J. Phys. Chem. 99 6242; b) Dittrich B, Hubschle C B, Luger P, Spackman M A 2006 Acta Cryst A62, 1325.

[18] Dominiak P, Espinosa E and Angyàn J G Intermolecular Interaction Energies from Experimental Charge Density Studies Modern charge density analysis (Springer 2012) ed C Gatti and P Macchi

[19] Jelsch C, Domagała S, Guillot B, Liebschner D, Fournier B, Pichon-Pesme V and Lecomte C Frontier Applications of Experimental Charge Density and Electrostatics to Bio-macromolecules Modern charge density analysis (Springer 2012) ed C Gatti and P Macchi.

[20] Dittrich B, Koritsanszky T and Luger P 2004 Angew Chem Int Ed Engl 432718.

[21] a) Zarychta B, Pichon-Pesme V, Guillot B, Lecomte C and Jelsch C 2007 Acta Cryst. A63 108; b) Domagała S and Jelsch C 2008 J. Appl. Cryst. 411140. 
[22] Volkov A, Li X, Koritsanszky T S and Coppens P 2004 J. Phys.Chem. A 1084283.

[23] Koritsanszky T, Volkov A and Chodkiewicz M 2012 Struc. \& Bond. 1471.

[24] Matta C F and Bader R W F 2000 Proteins-Structure Function And Genetics 40310.

[25] Guillot B, Jelsch C, Podjarny A and Lecomte C 2008 Acta Cryst. D64 567.

[26] Bak J M, Domagala S, Hubschle C B, Jelsch C, Dittrich B and Dominiak P M 2011 Acta Cryst A67 141.

[27] a) Jørgensen M R V, Cenedese S, Clausen H F, Overgaard J, Chen, Y-S, Gatti C and Iversen B B 2012 Inorg. Chem. 52 297; b) Poulsen R D, Bentien A, Chevalier M and Iversen B B 2005 J. Am. Chem. Soc. 127, 9156; c) Poulsen R D, Bentien A, Graber T and Iversen B B 2004 Acta Cryst. A60 382; d) Clausen H F, Overgaard J, Chen Y S and Iversen B B 2008 J. Am. Chem. Soc. 130, 7988; e) Jørgensen M R V, Clausen H F, Christensen M, Poulsen R D, Overgaard J and Iversen B B 2011 Eur. J. Inorg. Chem. 549; f) Meindl K, Henn J, Kocher N, Leusser D, Zachariasse K A, Sheldrick G M, Koritsanszky T and Stalke D 2009 J. Phys. Chem. A 113 9684.

[28] a) Spackman M A and Byrom P G 1997 Chem. Phys. Lett. 267 215; b) Spackman M A and Jayatilaka D 2009 CrystEngComm 1119.

[29] Hirshfeld F L 1977 Theor. Chim. Acta. 44129.

[30] Holladay A, Leung P C and Coppens P 1983 Acta Cryst. A39 377.

[31] Chimpri A S and Macchi P to be submitted.

[32] Bader R F W and Stephens M E 1974 Chem. Phys. Lett. 26445.

[33] Krawczuk-Pantula A, Pérez D, Stadnicka K and Macchi P 2011 Trans. Amer. Cryst. Ass. 1.

[34] a) Gadre S R and Shrivastava I H 1991 J. Chem. Phys. 94 4384; b) Bouhmaida N, Dutheil M, Ghermani N E and Becker P 2002 J. Chem. Phys. 116 6196; c) Mata I, Molins E, Alkorta I and Espinosa E 2007 J. Phys. Chem. 1116425.

[35]Zhu Q, Oganov A R, Salvado MA, Pertierra P, Lyakhov A O 2011 Phys. Rev. 83193410.

[36] Buckingham A D 1967 Adv. Chem. Phys. 12107.

[37] Spackman M A 1986 J. Chem. Phys. 856579.

[38] a) Cox S R, Hsu L-Y and Williams D E 1981 Acta Cryst. A37 293; b) Williams D E and Cox S R 1984 Acta Cryst. B40 404.

[39] Lennard-Jones J E 1924 Proc. R. Soc. Lond. A 106463.

[40] London F 1930 Z. Phys. 63245.

[41] Bertolasi V, Gilli P and Gilli G 2012 Crystal Growth \& Design 124758.

[42] Schroeder R and Lippincott E R 1955 J. Chem. Phys. 231099.

[43] Silvi B, Gatti C 2000 J. Phys. Chem. 104947.

[44] Volkov A, Koritsanszky T and Coppens P 2004 Chem. Phys. Lett. 391, 170.

[45] Keskin O, Gursoy A, Ma B and Nussinov R 2008 Chem. Rev. 1081225.

[46] Rowsell J L C, Spencer E C, Eckert J, Howard J A K, Yaghi O M 2005 Science 3091350.

[47] Volkov A, Macchi P, Farrugia L J, Gatti C, Mallinson P, Richter T and Koritsanszky T XD2006 - A Computer Program Package for Multipole Refinement, Topological Analysis of Charge Densities and Evaluation of Intermolecular Energies from Experimental and Theoretical Structure Factors. University of Buffalo 2006.

[48] Dunmur D A 1972 Mol. Phys. 23109.

[49] Whitten A E, Jayatilaka D and Spackman M A 2006 J. Chem. Phys. 125174505.

[50] Chimpri A S, Gryl M, Dos Santos L H R, Krawczuk A and Macchi P, submitted.

[51] Cummins P G, Dunmur D A, Munn R W and Newham R J 1976 Acta Cryst. A32 854. 